\title{
Mechanisms of Water Catalysed Reactions
}

\section{Eduardo Humeres}

Departamento de Química, Universidade Federal de Santa Catarina, 88040-970, Florianópolis, SC, Brazil

\section{Water Catalyzed Reactions}

Water is unique among liquids for its ability for tetrahedral coordination with four neighbouring molecules. Water catalyzed reactions (or spontaneous reactions) are $\mathrm{pH}$ independent hydrolytic processes that involve the transfer of one or several protons to or from water molecules in the transition state (TS) of the rate determining step. Indeed, in these reactions one water molecule acts as a nucleophile while one or several act as general bases, and typically show a) high negative values of entropy of activation $\left(\Delta S^{\neq}\right)$, and b) high kinetic solvent isotope effect (KSIE). In these reactions water is solvent, nucleophile and catalyst. The hydrolyses of alkyl halides, alkyl and aryl sulphonates, and derivatives of carboxylic acids ( esters, amides) have been extensively studied. The reaction with derivatives of saturated carbons is quite different than the hydrolyses of esters and amides. At least two water molecules must be strongly bound at the TS to produce a high KSIE, restricting the number of possible positions of these molecules. The number of molecules involved in the proton transfer ( two molecules per proton) can be determined by proton inventory. Polymolecular mechanisms have been found for these reactions with 3-5 molecules involved in the TS. Hydrophobicity and hydrophilicity of the substrate is determinant of the mechanism.

\section{Supramolecular Catalysis Induced by Polysaccharides}

The non-bonding interactions of carbohydrates with water depend on their stereochemistry. Kinetic medium effects induced by carbohydrates are important to the understanding of their role in the sugarprotein recognition involved in carbohydrate transport, the relationship antigen-antibody of the immunological system and hydrolytic enzymatic reactions. Monosaccharides, depending on their stereochemistry, inhibit specifically water catalyzed reactions of small molecules [1] while modified polysaccharides induce water molecules into a highly ordered supermolecular structure that can then catalyze a reaction on the polysaccharide matrix.

In 1991 it was observed that the water catalyzed reaction of a cellulose xanthate ester was about 2000 times faster than the small analogue molecule and it was proposed that the acceleration was a consequence of the highly ordered cybotactic region and that consequently $\Delta S^{\neq}$should be nearly zero [2]. This assumption was confirmed in a detailed study of the water catalyzed hydrolysis of p- 
nitrobenzyl cellulose xanthate (CelXNB). The rate determining step was the nucleophilic attack of a water molecule [3], catalyzed by a second water molecule that acts as a general base [4]. The water catalysis is not due to a neighboring $\mathrm{OH}$ effect [5] and $\Delta \mathrm{S}^{\neq}$is nearly zero $\left(+3.6 \mathrm{cal} \cdot \mathrm{mol}^{-1} \cdot \mathrm{K}^{-1}\right)$. The spontaneous hydrolysis of 2,4-dinitrophenyl cellulose xanthate in acetone-water mixtures confirmed that the hydrolysis does not occur through water polymers and that above $30 \mathrm{M}$ there are no acetone molecules (or very few) in the highly-ordered cybotactic region of cellulose (selective solvation) [5].

\section{Intramolecular Proton Transfer and Torsional Effect}

The cleavage of aryl and some alkyl dithiocarbamates occurs through a water catalyzed intramolecular $\mathrm{S}$ to $\mathrm{N}$ proton transfer concerted with the $\mathrm{C}-\mathrm{N}$ bond cleavage [6]. Theoretical ab initio calculations supported this hypothesis. The driving force to reach the TS is the torsional effect of the $\mathrm{C}-\mathrm{N}$ bond that inhibits the resonance with the thiocarbonyl group increasing the basicity of the nitrogen and making the proton transfer thermodynamically favorable.

\section{References and Notes}

1. (a) Galema, S.A.; Blandamer, M.J.; Engberts. J.B.F.N. J. Am. Chem. Soc. 1990, 112, 9665; (b) Galema, S.A.; Blandamer, M.J.; Engberts, J.B.F.N. J. Org. Chem. 1992, 57, 1995.

2. Humeres, E.; Oliveira, C.M.S.; Osellame, V.T.; de Souza, I. J. Phys. Org. Chem. 1991, 4, 573.

3. Humeres, E.; Sequinel, L.F.; Nunes, M.; Oliveira C.M.S.; Barrie, P.J. J. Phys. Org. Chem. 1994, $7,287$.

4. Humeres, E.; Sequinel, L.F.; Nunes, M.; Oliveira C.M.S.; Barrie, P.J. Can. J. Chem. 1998, 76, 960.

5. Humeres, E.; Soldi, V.; Klug, M.; Nunes, M.; Oliveira, C.M.S.; Barrie, P. J. Can. J. Chem. 1999, $77,1050$.

6. Humeres, E.; Debacher, N.A.; de S. Sierra, M.M.; Franco, J.D.; Schutz, A. J. Org. Chem. 1998, 63, 1598. 